Reyes Fernández, B., Montenegro-Montenegro, E., Knoll, N., Schwarzer, R. (in press). Self-efficacy, Action Control, and Social Support Explain Physical Activity Changes among Costa Rican Older Adults. Journal of Physical Activity and Health 04/2014; DOI:10.1123/jpah.2013-0175

\begin{abstract}
Background: Self-efficacy, action control, and social support are considered to influence changes in physical activity levels in older adults. This study examines the relationship among these variables and explores the putative mediating and moderating mechanisms that might account for activity changes.
\end{abstract}

Methods: A longitudinal study with 54 older adults ( $\geq 50$ years of age) was carried out in Costa Rica. In a moderated mediation analysis, action control was specified as a mediator between self-efficacy and physical activity, whereas social support was specified as a moderator between self-efficacy and action control. Baseline physical activity, age, and sex were specified as covariates.

Results: Action control mediated between self-efficacy and physical activity. An interaction between social support and self-efficacy on action control pointed to a synergistic effect at the first stage of the mediating process.

Conclusions: The effect of self-efficacy on physical activity was partly explained by action control, providing evidence of action control as a proximal mediator of physical activity. Moreover, the moderator role of social support was confirmed: high social support appeared to compensate for low levels of self-efficacy. 


\section{Self-efficacy, Action Control, and Social Support Explain Physical Activity Changes among Costa Rican Older Adults}

As the population becomes older, more attention is being paid to physical activity as a strategy to increase quality of life and facilitate healthy aging. Moderate but regular physical activity is related to multiple health benefits such as the reduction in mortality rates, prevention of some kinds of cancers, and reduced risk of developing dementia, among others ${ }^{1-}$ ${ }^{4}$. In Costa Rica, $28 \%$ of older adults are reported to be physically active ${ }^{5}$, but there is no specific information about the frequency and the intensity of physical activity. Considering the benefits of physical activity on health, it is recommended to increase the number of Costa Rican older adults who practice physical activity. A better understanding of the psychological process to initiate and maintain physical activity would facilitate health promotion. Various theoretical models provide frameworks to specify which psychosocial variables might serve to explain and promote physical activity ${ }^{6}$. Previous research on perceived self-efficacy and on social support also broadens the understanding of physical activity changes in older adults ${ }^{7-9}$.

\section{Perceived self-efficacy as a facilitator of behavior change}

Self-efficacy is a consistent predictor of physical activity initiation and maintenance . $^{7}$

Research among older adults is no exception to this, and self-efficacy has been found to exert direct and indirect effects on physical activity in seniors ${ }^{10}$. Self-efficacy portrays individuals' beliefs in their capabilities to perform a specific action required to attain a desired outcome. It can be characterized as being competence-based, prospective, and action-related ${ }^{11}$. These beliefs on control over challenging demands are critical in approaching novel or difficult behavior, or in adopting a strenuous self-regimen. Put differently, self-efficacy might be 
critical to tackle demanding tasks and to cope with challenges that may become stressful at some point of time. Self-efficacy influences the decisions that people make as well as how high they set their goals (e.g., "I intend to be more physically active on a regular basis starting tomorrow"). If people believe that they can take action to solve a problem instrumentally, such as beginning to enhance daily physical activity by going swimming every morning even though this requires complex preparatory behavior, they become more inclined to do so and feel more committed to this decision. Within the framework of the Health Action Process Approach two phases of behavior change are specified, namely, a motivational and a volitional phase ${ }^{6}$. Self-efficacy plays an important role in both phases. In the first phase of the process, in which an individual does not yet act, but develops a motivation to do so, selfefficacy represents the pre-actional confidence in one's capabilities to perform a challenging behavior. As a motivational variable, it is expected to precede volitional variables such as action control that become relevant at a later stage when people try to act according to their behavioral intentions.

\section{Action control as a proximal predictor of behavioral maintenance}

The effect of self-efficacy on physical activity needs to be further clarified, because not everyone with a high level of self-efficacy actually performs the intended behavior. How does self-efficacy exert its effect on physical activity? Mediating mechanisms may be responsible for the effect of self-efficacy on behavior change. Action control has been found to work as a mediator between self-efficacy and exercise ${ }^{12}$. Action control is considered to be a proximal predictor of health behavior and is related to the concept of a self-regulatory feedback loop. Action control processes include focusing one's attention on the task, while avoiding attention to distractors, resisting temptations, and managing unpleasant emotions. 
The concept of action control is based on self-regulation theory ${ }^{13}$. It represents a feedback loop, where there is (a) an input value generated by an information collection function (e.g., "I went swimming on Monday and Wednesday."); (b) a standard value (e.g., "I intend to go swimming every day."); (c) a comparator analyzing differences between input and standard (e.g., "I missed one day of swimming"); and (d) an output function aiming at reducing differences between input and standard (e.g., "I will make sure not to miss another day of swimming"). These cognitive processes can be summarized into the three facets of action control, namely, awareness of standards (goals), self-monitoring of progress, and selfregulatory effort. Action control can mediate the influence of several predictors on behavior, such as intentions, planning, and self-efficacy ${ }^{12}$.

\section{The moderator role of social support: Who benefits from it?}

Thus, whereas the effect of self-efficacy on behavior might be explained through action control, this effect might not be equally effective for everybody. Within the research context of health behavior change, moderator variables have been identified ${ }^{14,15}$. In other words, at one level of a moderator variable the outcome is predicted well, but at another level this is not the case. Thus, a moderator answers the question for whom an effect applies and for whom it does not.

Social support contributes to explain health behavior change ${ }^{8,9,16,17}$. It represents a resource factor, among others, that influences the cognitive appraisal of stressful encounters and facilitates coping. It refers to a function of social relationships, such as perceived availability of help or support actually received. Support occurs through an interactive process and can be related to altruism, a sense of obligation, and the perception of reciprocity ${ }^{18-20}$. Social support may involve specific instances of actual support (received support) ${ }^{18}$ or merely the perception 

of support available in case of need (perceived support). In the present study, the focus was on actual circumstances and not on mere expectations. Therefore received support was chosen ${ }^{18}$. As a social coping resource, it may operate in concert with other resource factors such as selfefficacy.

\section{Relationship between self-efficacy, action control, social support, and physical activity.}

When looking for changes in physical activity, the most obvious predictor is perceived selfefficacy. This is based upon a large body of empirical evidence ${ }^{7,10,11}$. The roles of action control and social support in this context are intuitive but less obvious. To study the operative mechanisms among these three variables in terms of predicting physical activity in middle aged to old individuals, around the age of retirement, one might look at more comprehensive self-regulation theories as individuals in these later parts of the life span might already begin to face additional health-related barriers that increase the difficulty of behavior change ${ }^{13}$. Among self-regulatory variables, an interplay can be expected which leads to feedback processes toward a desired goal. This feedback might come from social sources. A possible mechanism is the following. Self-efficacy leads individuals to confront difficult challenges. By this, self-efficacy can contribute to stimulate action control, first, by making people invest their cognitive resources in setting a behavioral goal and related standards, and second, by fostering self-monitoring strategies to assess the discrepancy between their actual performance and the intended one, and last, by increasing the likelihood to reduce this discrepancy through self-regulatory efforts. But when self-efficacy is not high enough to incite action control, social support might act as an external source of motivation and information to let action control increase. Network partners providing support to individuals 
Running head: Physical Activity Changes among Costa Ricans who try to increase regular physical activity might act as additional sources of social regulation inspiring close monitoring and optimization of behavior change in the acting persons. Thus, social support could then compensate for low levels of self-efficacy to cope with the stressful demands which come after an intention is set and, in this way, act as a buffer. However, so far, little has been found on the relationship between social support and action control.

\section{Aims}

The aim of the present investigation is to examine the predictive value of self-efficacy, action control, and social support on physical activity changes in older adults in Costa Rica. Another question addressed whether the effects of self-efficacy on physical activity can be explained by action control and whether there is an interaction effect between self-efficacy and received social support on the levels of action control. Put differently, the role of social support on a motivational variable (self-efficacy) and a volitional variable (action control) is examined. This leads to the following hypotheses:

1. Self-efficacy predicts physical activity. Self-efficacy predicts action control as well, because it contributes to set standards, to implement self-monitoring strategies, and to make an effort to reduce the discrepancy between actual and intended behavior.

2. Action control predicts physical activity and explains the effect of self-efficacy on physical activity. This constitutes a mediation effect of action control between selfefficacy and physical activity.

3. Self-efficacy predicts action control and, given that action control is also predicted by social support, there might be an interaction between these two predictors. This could 
mean that social support moderates the relationship between self-efficacy and action control. Individuals with lower levels of self-efficacy might then benefit more from received social support than people with higher self-efficacy. Put differently, low social support might be unfavorable for individuals with lower self-efficacy, but for persons with higher self-efficacy the levels of support might not make a difference with regard to their action control.

\section{Methods}

\section{Participants and procedure}

Participants were recruited for a longitudinal study in two educational programs in Costa Rica. These programs, held by the University of Costa Rica and the Costa Rican Gerontological Association, offer courses for men and women of 50 years or older. Invitations were delivered in the classrooms, and 447 persons provided data to be contacted. Of them, 166 filled out the questionnaire at Time 1 (T1). Three weeks later, participants were invited again by a telephone call, and 54 individuals completed the Time 2 (T2) assessments. Before filling out the $\mathrm{T} 1$ questionnaires, the participants provided informed consent, as required by the Ethics Committee of the University of Costa Rica.

Of the longitudinal sample at T2, most of the participants were women (75.9\%), most had complete or incomplete university studies $(70.3 \%)$, and their mean age was 65.3 years $($ range $=50-85$ years, S.D. $=7.74)$. 
Running head: Physical Activity Changes among Costa Ricans

\section{Measures}

With the only exception of physical activity frequency and socio-demographic variables (sex, age, and education), all variables were measured with four-point Likert scales. Item examples are translations from Spanish. Table 1 presents the complete set of items for self-efficacy, social support and action control.

Self-efficacy was measured at Time 1 by a scale composed of three items (Cronbach's $\alpha=$ $.69)^{12}$. An example item is "I am confident that I can practice physical activity on a regular basis".

At Time 1, received social support was measured with two items (Cronbach's $\alpha=.80)^{9}$. An example item is "There was someone who encouraged me to practice physical activity on a regular basis".

The putative mediator action control was measured at Time 2 with six items (Cronbach's $\alpha=$ $.91)^{12}$. An example item is "I have kept frequently in mind the intention of being physically active".

At Times 1 and 2, frequency of moderate physical activity was measured with an instrument ${ }^{9}$, asking “on how many days of the last week have you practiced moderate physical activity?". Answers could range from 1 to 7 . Means, intercorrelations, and reliability of the variables are shown in Table 2. 
Table 1. Items for self-efficacy, action control and social support.

\begin{tabular}{|c|c|}
\hline Variable & Items \\
\hline $\begin{array}{l}\text { 1. Self- } \\
\text { efficacy }\end{array}$ & $\begin{array}{l}\text { 1) I am confident that I can practice physical activity on a regular basis. } \\
\text { 2) I am confident that I can practice physical activity in the long run. } \\
\text { 3) I am confident that I can practice physical activity some minutes during the next } \\
\text { weeks. }\end{array}$ \\
\hline $\begin{array}{l}\text { 2. Social } \\
\text { support }\end{array}$ & $\begin{array}{l}\text { 1) Someone encouraged me to practice physical activity on a regular basis. } \\
\text { 2) I felt support to practice physical activity on a regular basis. }\end{array}$ \\
\hline $\begin{array}{l}\text { 3. Action } \\
\text { Control }\end{array}$ & $\begin{array}{l}\text { 1) I have constantly monitored if I practice enough physical activity. } \\
\text { 2) I have carefully observed that I have trained at least } 30 \text { minutes with the } \\
\text { recommended strain for each occasion. } \\
\text { 3) I have had frequently in mind the intention of being physically active. } \\
\text { 4) I have been always aware of my plan to remain physically active. } \\
\text { 5) I have tried to exercise regularly. } \\
\text { 6) I have tried to give my best to act in accordance to my standards. }\end{array}$ \\
\hline
\end{tabular}

\section{Analytical Procedure}

A mediated moderation analysis was carried out using the SPSS Process macro by Hayes ${ }^{21}$. As a first step, the putative mediator (action control) is regressed on the independent variable (self-efficacy) and on the putative moderator (social support) as well as on their interaction term (self-efficacy x social support). The second step consists of the regression of the dependent variable (physical activity) on the independent variable (self-efficacy), on the putative mediator (action control), and on covariates. Estimates of all paths were calculated by ordinary least squares procedures. The number of bootstrapped resamples was 5,000, yielding $95 \%$ confidence intervals of the parameter estimates. 
Running head: Physical Activity Changes among Costa Ricans

\section{Results}

\section{Attrition analysis}

Differences between 112 participants who dropped out from T1 (67\% of the original sample) and those who continued at T2 $(\mathrm{N}=54)$ were examined. Participants who dropped out indicated lower self-efficacy levels than those who completed the second measurement (for self-efficacy: MAttrition $=2.57 S D=0.55 ;$ MCompleters $=2.76 S D=.36, p=.02)$. No differences between groups were found for social support $(p=.57)$ and baseline frequency of physical activity $(p=.47)$.

\section{Descriptive statistics}

The means, standard deviations, and reliabilities for each study variable, as well as the intercorrelations among them, are presented in Table 2. 
Table 2. Descriptive statistics, reliability and intercorrelations for self-efficacy ( $T 1$ ), social support (T1), action control (T2), frequency of moderate physical activity at T2, and frequency of moderate physical activity at T1.

\begin{tabular}{lccccc}
\hline Variable & 1 & 2 & 3 & 4 & 5 \\
\hline Cronbach's a & .69 & .80 & .91 & - & - \\
Mean & 2.76 & 1.33 & 3.61 & 2.55 & 2.35 \\
SD & 0.36 & 0.59 & 0.63 & 1.97 & 2.16 \\
1. Self-efficacy T1 & 1 & & & & \\
2. Social support T1 & -.13 & 1 & & & \\
3. Action control T2 & $.49^{* *}$ & .19 & 1 & & \\
4. Frequency of moderate physical & .12 & -.05 & $.42^{* *}$ & & \\
$\quad \begin{array}{l}\text { activity T2 } \\
\text { 5requency of moderate physical }\end{array}$ & .07 & -.25 & $.38^{* *}$ & $.71^{* *}$ \\
$\quad$ activity T1 & & & & & 1 \\
\hline
\end{tabular}

Note: ${ }^{* \star} p<.01$.

\section{Mediated moderation analysis}

Figure 1 shows the main results. As a first step, the mediator action control (T2) was regressed on self-efficacy (T1), social support (T1) and the interaction between social support and self-efficacy. Action control was predicted by both, social support and self-efficacy, as well as by their interaction term. In a second step, the frequency of physical activity (T2) was regressed on the independent variable self-efficacy (T1), the mediator action control (T2), as well as on the three covariates baseline physical activity, age, and sex (all T1). The indirect effect of self-efficacy on physical activity through action control was found to fall within the bootstrapped confidence intervals, $95 \%$ at $-1 \mathrm{SD}$ of social support $(b=1.21, C I[0.26,3.01])$, at the mean $(b=.78, C I[0.19,1.70])$, and at $+1 \mathrm{SD}$ of social support $(b=.35, C I[0.08$, 1,10]). After controlling for action control, the direct relationship between self-efficacy and 

physical activity became non-significant $(p=.55)$. In other words, self-efficacy seemed to exert its effect on physical activity through action control, which had a significant relationship with the outcome variable. Actually, the stronger predictor of T2 physical activity was action control, followed by baseline physical activity. Among the three covariates specified in the model, only baseline physical activity was statistically significant whereas sex and age did not make a contribution. Overall, the variables of the model accounted for $R^{2}=.59$ of the variance in physical activity.

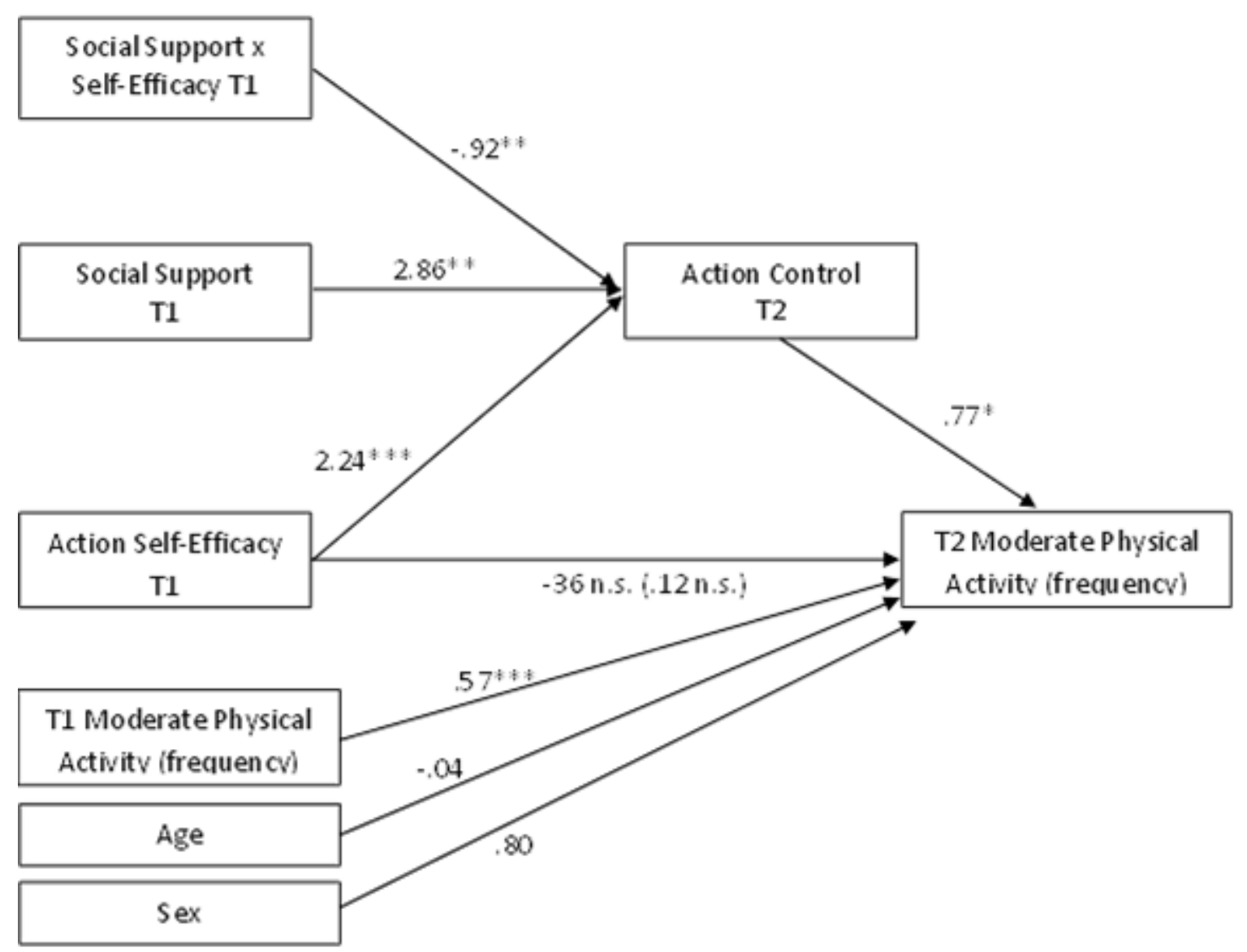

Figure 1. Mediated moderation model for physical activity. Note: ${ }^{*} p<.05, * * p<.01, * * * p<.001 ;$ men $=1$, women $=2$. Direct effect (c path) is in parentheses. Parameters are expressed in unst andardized values. Control variables we re baseline physical activity, age and sex.

The interaction between self-efficacy and social support is illustrated in Figure 2. Higher levels of self-efficacy corresponded with higher levels of action control. However, the effect 

size of self-efficacy on action control decreased and became less significant as social support increased. For instance, when social support was $1 S D$ higher $(b=1.93)$ than the mean, the effect was only marginal $(p=.08)$. The Johnson-Neyman technique provides a more detailed view, showing that the cut-off point from which self-efficacy no longer predicted action control is when the social support score attains $b=1.89$.

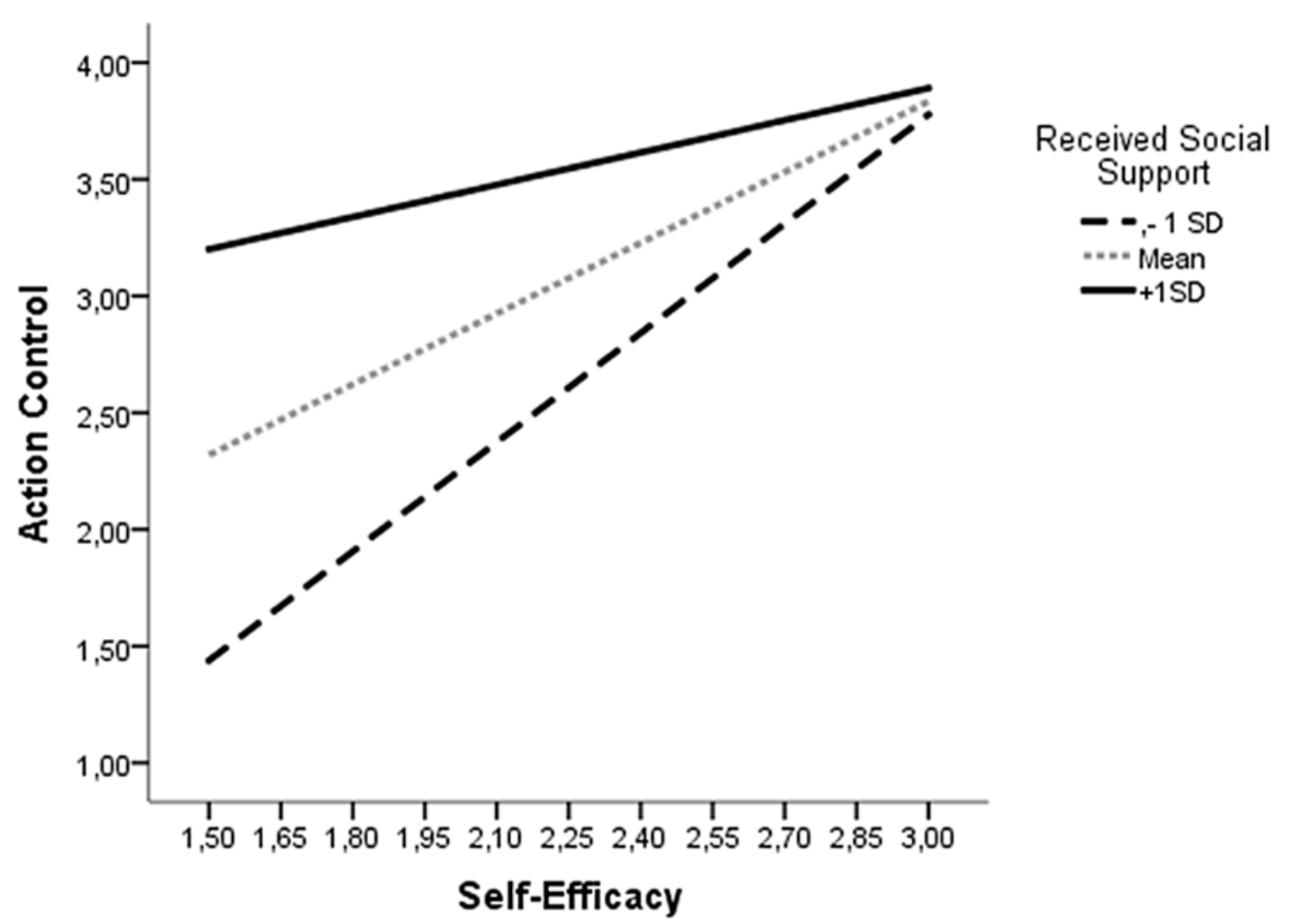

Figure 2. Moderation of social support Time 1, between self-efficacy Time 1 and action control Time 2.

\section{Discussion}

This study has explored the possible social-cognitive mechanisms that play a role in physical activity changes in middle-aged to old Costa Rican adults. Previous evidence suggests that self-efficacy is a consistent determinant of physical activity, but other self-regulatory 
Running head: Physical Activity Changes among Costa Ricans variables contribute to this prediction in some way ${ }^{6,7,10}$. As promising candidates for other constructs we have chosen action control $^{12}$ and social support ${ }^{9}$.

The findings of the present study provide support for each of the hypotheses proposed. Selfefficacy predicted the frequency of moderate physical activity, however, not directly but rather via action control. To be confident in one's capabilities might not be enough to perform certain behaviors. One has to implement a strategy to be aware of the standards related to the intended behavior, one has to pay attention to the actual behavior being performed, and then, after comparing current behavior with the standards, one has to make an effort to reduce the discrepancy. This constitutes evidence for the first of the hypotheses proposed.

The importance of action control became apparent, given its strength to predict behavior. No other single predictor had a stronger effect on physical activity. Action control mediated the effect of self-efficacy on frequency of moderate physical activity, which supported the second hypothesis.

Social support also increased levels of action control. Thus, a self-regulatory strategy such as action control can benefit from both resources, self-efficacy as well as social support. The positive relationship between self-efficacy and action control was more pronounced among older adults with lower levels of social support. This constitutes evidence for the third hypothesis proposed, underscoring that when self-efficacy is low, social support can compensate for it when it comes to predict action control. For individuals with lower selfefficacy, low social support is detrimental when it comes to action control. But for persons with higher self-efficacy, their levels of support do not make a difference with regard to their action control. 
Running head: Physical Activity Changes among Costa Ricans Previous research ${ }^{9}$ has focused on the joint effects of self-efficacy and social support directly on behavior, but not on self-regulatory strategies such as action control. The effects of selfregulatory variables as mediators between resources and behavior need to be further examined, and the present study makes a contribution in this direction.

The results show that social support interacts with a motivational variable (self-efficacy) exerting an effect on a volitional variable (action control), which underscores the relevance of social support as a moderator in the health behavior change process.

Implications of these findings should be pointed out. Strategies to increase action control levels could be included in intervention programs, because this works as an influential proximal variable. Interventions could include an explicit focus on self-monitoring the actual level and frequency of one's ongoing activity and possible discrepancies to one's goals.

The interplay between self-efficacy and social support should also be taken into account. Low self-efficacious as well as poorly supported individuals should be identified before an intervention in order to improve at least one of these two coping resources.

Some limitations need to be mentioned. Because there was no experimental research design, causal inferences cannot be made. However, the proposed model is theoretically founded and the placement of the variables in a longitudinal design suggests directional conclusions. The main problem, however, is the high drop-out rate leading to a small remaining sample. On the other hand, it is noteworthy that the effects were strong enough to become significant in spite of the small sample size.

The use of self-reports for assessing behavior can be criticized as well, but this is in line with most of the previous literature, and it is generally assumed that self-reports of physical activity are valid in a satisfactory manner ${ }^{22}$. 
Running head: Physical Activity Changes among Costa Ricans Despite these limitations, the findings point to the interplay of self-regulatory mechanisms with social exchange processes when it comes to changing physical activity levels in middleaged and old adults and, thus, the findings may contribute to the emerging literature on this issue.

In the Costa Rican context, analyses based on longitudinal data on physical activity among older adults represent an advantage over the usually available cross-sectional data on which local health promotion policies rely. Further longitudinal and intervention studies need to be conducted to provide reliable information on which to base public health policies. Although theoretical models have proven utility to explain physical activity in different countries, further research might be useful to help local authorities and professionals to make informed decisions. 


\section{References}

1. Vogel T, Brechat PH, Leprêtre PM, Kaltenbach G, Berthel M, Lonsdorfer J. Health benefits of physical activity in older patients: a review. International Journal of Clinical Practice. 2009;63(2):303-320.

2. Hardman AE. Physical activity and cancer risk. Proc. Nutr. Soc. Feb 2001;60(1):107113.

3. Wendel-Vos GCW, Schuit AJ, Feskens EJM, et al. Physical activity and stroke. A meta-analysis of observational data. Int. J. Epidemiol. Aug 2004;33(4):787-798.

4. Larson EB, Wang L, Bowen JD, et al. Exercise is associated with reduced risk for incident dementia among persons 65 years of age and older. Ann. Intern. Med. Jan 2006;144(2):73-81.

5. UCR/CONAPAM. I Informe del Estado de Situación de la Persona Adulta Mayor en Costa Rica. 2008.

6. Schwarzer R. Modeling health behavior change: How to predict and modify the adoption and maintenance of health behaviors. Appl Psychol-Int Rev. Jan 2008;57(1):1-29.

7. van Stralen MM, De Vries H, Mudde AN, Bolman C, Lechner L. Determinants of initiation and maintenance of physical activity among older adults: a literature review. Health Psychology Review. 2009/09/01 2009;3(2):147-207.

8. Gellert P, Ziegelmann JP, Warner LM, Schwarzer R. Physical activity intervention in older adults: does a participating partner make a difference? Eur J Ageing. Sep 2011;8(3):211-219.

9. Warner LM, Ziegelmann JP, Schuz B, Wurm S, Schwarzer R. Synergistic effect of social support and self-efficacy on physical exercise in older adults. $J$ Aging Phys Activ. Jul 2011;19(3):249-261.

10. White SM, Wójcicki TR, McAuley E. Social cognitive influences on physical activity behavior in middle-aged and older adults. The Journals of Gerontology Series B:

Psychological Sciences and Social Sciences. January 1, 2012 2012;67B(1):18-26.

11. Bandura A. Self-efficacy: The exercise of control. New York: Freeman; 1997.

12. Sniehotta FF, Scholz U, Schwarzer R. Bridging the intention-behaviour gap: Planning, self-efficacy, and action control in the adoption and maintenance of physical exercise. Psychol Health. Apr 2005;20(2):143-160.

13. Carver CS, Scheier M. On the self-regulation of behavior. Cambridge, UK ; New York, NY, USA: Cambridge University Press; 1998.

14. Evers A, Klusmann V, Schwarzer R, Heuser I. Adherence to physical and mental activity interventions: Coping plans as a mediator and prior adherence as a moderator. Brit J Health Psych. Sep 2012;17:477-491. 
15. Parschau L, Fleig L, Koring M, et al. Positive experience, self-efficacy, and action control predict physical activity changes: A moderated mediation analysis. Brit J Health Psych. 2013;18(2):395-406.

16. Molloy GJ, Dixon D, Hamer M, Sniehotta FF. Social support and regular physical activity: Does planning mediate this link? Brit J Health Psych. Nov 2010;15:859-870.

17. Scholz U, Ochsner S, Hornung R, Knoll N. Does social support really help to eat a low-fat diet? Main effects and gender differences of received social support within the Health Action Process Approach. Applied Psychology: Health and Well-being. 2013;5:270-290.

18. Schwarzer R, Knoll N. Functional roles of social support within the stress and coping process: A theoretical and empirical overview. Int J Psychol. Aug 2007;42(4):243252.

19. Schwarzer R, Knoll N. Social Support. In: French D, Kaptein A, Vedhara K, Weinman J, eds. Health Psychology. 2nd ed. Oxford, UK.: Wiley-Blackwell; 2010:283-293.

20. Knoll N, Burkert S, Roigas J, Gralla O. Changes in reciprocal support provision and need-based support from partners of patients undergoing radical prostatectomy. Soc Sci Med. Jul 2011;73(2):308-315.

21. Hayes AF. PROCESS: A versatile computational tool for observed variable mediation, moderation, and conditional process modeling. . 2012. http://www.afhayes.com/public/process2012.pdf.

22. Prince SA, Adamo KB, Hamel ME, Hardt J, Gorber SC, Tremblay M. A comparison of direct versus self-report measures for assessing physical activity in adults: a systematic review. Int J Behav Nutr Phy. Nov 6 2008;5. 